\title{
REES CONGRUENCES ON RESIDUATED SEMIGROUPS
}

\author{
C. NKUIMI-JUGNIA and A. OGADOA \\ Department of Mathematics \\ Faculty of Sciences \\ University of Yaounde \\ Cameroon \\ e-mail: cnkuimi@gmail.com \\ cogadoaamassayoga@yahoo.fr
}

\begin{abstract}
Residuated semigroups form an important class of ordered algebra which can regarded a generalisation of quantales. We study the notion of quotient on a residuated semigroup extending some results of [27] to them. A particular attention is put on Rees congruences on residuated semigroups.
\end{abstract}

\section{Introduction and Preliminaries}

Residuation is a basic concept in mathematics [2]. It is strongly connected with Galois connections and closure operators. Residuated semigroups have been introduced in the 30 s of the last century by Ward and Dilworth [26] to investigate ideal theory of commutative rings with unit. Nowadays, the investigation of residuated lattices (that is, residuated monoids on lattices) has become quite popular, and has been staying in the focus of strong international attention. An extensive 
monograph discussing residuated lattices went to print in 2007 [9]. This increasing and significant interest was initiated by the discovery of the strong connection between residuated lattices and substructural logics $[18,19]$. Residuated semigroups constitute a vast class of ordered algebras comprising both residuated lattices and quantales. This paper deals with quotient of residuated semigroups; it extends some results of [27].

An ordered semigroup is an ordered set $(P, \leq)$ together with semigroup structure $(\otimes: P \times P \rightarrow P)$ such that the order $\leq$ that is compatible with the semigroup operation, meaning that $x \leq y$ implies $z \otimes x \leq z \otimes y$ and $x \otimes z \leq y \otimes z$ for all $x, y, z \in P$. Ordered monoid are defined analogously. If $P$ is a group and it is ordered as a semigroup, one obtains the notion of ordered group. The terms posemigroup, pogroup, and pomonoid are also in use.

Definition 1.1. A partially ordered semigroup $(P, \leq, \otimes)$ is called residuated if all left translation $P \stackrel{a \otimes-}{\longrightarrow} P$ and all right translations $P \stackrel{-\otimes a}{\longrightarrow} P$ are residuated, i.e., have right adjoints $P \stackrel{a \backslash-}{\longrightarrow} P$ and $P \stackrel{-/ a}{\longrightarrow} P$, respectively; this means that

$$
a \otimes x \leq y \text { and } x \leq a \backslash y
$$

are equivalent condition; similarly for

$$
x \otimes a \leq y \text { and } x \leq y / a .
$$

Residuated po-semigroups will be simply be called residuated semigroups.

Clearly,

$$
a \leq b \text { implies } b \backslash x \leq a \backslash x \text { and } x \backslash a \leq x \backslash b \text {. }
$$

It follows from (1), (2), and (3) that $a \leq b /(a \backslash b)$, with equality if and only if $a=b / x$, for some $x$ in $P$. 
The two binary operations $P \times P \stackrel{-\backslash-}{\longrightarrow} P$ and $P \times P \stackrel{-/-}{\longrightarrow} P$ are called the left and the right residuals of $\otimes$. More precisely $c / b$ is called the right residual of $c$ over $b$, and $b \backslash c$ the left residual of $c$ over $b$.

Definition 1.2. If $P$ and $Q$ are residuated semigroups, then a mapping $F: P \rightarrow Q$ is a residuated semigroup homomorphism if and only if it preserves the multiplication $\otimes$ and the two residuals $-\backslash-$ and $-/-$.

A residuated po-monoid for which the order $\leq$ is the lattice order is called residuated reticulated monoid or simply a residuated lattice. Examples of residuated lattices include Boolean algebras, Heyting algebras [12], MV-algebras [4], basic logic algebras, [11] and lattice-ordered groups. Complete residuated semigroups are referred to as quantales and complete residuated monoid are referred to as unital quantales.

Example 1.3 (Powerset residuated semigroup). Let $(M, \cdot)$ be a semigroup. For two subsets $X$ and $Y$ of $M$, we define

$$
\begin{gathered}
X \otimes Y=\{x \cdot y: x \in X \text { and } y \in Y\}, \\
X \backslash Y=\{z \in M: X \otimes\{z\} \subseteq Y\},
\end{gathered}
$$

and

$$
Y / X=\{z \in M:\{z\} \otimes X \subseteq Y\} .
$$

The structure $\left(2^{M}, \subseteq, \otimes, \backslash, /\right)$ is a residuated semigroup. It is called the powerset residuated semigroup over the semigroup $(M, \cdot)$. This example is of importance in the theory of languages where $M$ is usually the free semigroup over an alphabet.

Example 1.4. Let $E$ be a set and let's denote by $\operatorname{Rel}(E)$ the set $\mathcal{P}(E \times E)$ of relations on $E$. One gets the residuated monoid $\left(\operatorname{Rel}(E) ; \subseteq, \otimes, \backslash, /, \Delta_{E}\right)$, where $\Delta_{E}$ denotes the equality relation on $E$ and $R \otimes S$ the compositional relation of the relations $R$ and $S$ 
(alternative notation is $S \circ R$ ). The right residuate $S / R$ of $S$ over $R$ is $\{(x, y), \forall z[y R z \Rightarrow x S z]\}$; the left residuate $R / S$ of $S$ over $R$ is $\{(y, z)$, $\forall x[x R y \Rightarrow x S z]\}$.

Example 1.5. Let $R$ be a ring with unit and let $\operatorname{Ideals}(R)$ be the set of all (two-sided) ideals of $R$ equipped with set inclusion. For $I, J \in$ $\operatorname{Ideals}(R)$, we write $I \otimes J=\left\{\sum_{\text {finite }} i j: i \in I, j \in J\right\}, \Gamma J=\{k: I \otimes\{k\}$ $\subseteq J\}, J / I=\{k:\{k\} \otimes I \subseteq J\}$. One gets the residuated monoid (Ideals $(R)$, $\subseteq, \otimes, \backslash, /, R)$.

\section{Regular Congruences on Residuated Ordered Semigroups}

For an equivalence relation $\theta$ a set $P$ we denote by $\theta^{b}(x)$ the equivalence class of $x$ and therefore by $\theta^{b}: P \rightarrow P / \theta$ the canonical surjection. If $\theta$ be a congruence on a universal algebra $\mathbf{P}=(P, \otimes)$, the quotient semigroup (also known as the homomorphic image) $\mathbf{P} / \theta$ of $\mathbf{P}$ by $\theta$ has support the quotient set $P / \theta$ of $\theta$-classes and its multiplication, again denoted by $\otimes$, is given by:

$$
\theta^{b}(x) \otimes \theta^{b}(y):=\theta^{b}(x \otimes y) .
$$

It is known how to extend the concept of quotient to po-semigroup. Let us first quote the following definition:

Definition 2.1 ([23]). Let $(P, \leq, \otimes)$ be a po-semigroup, $\theta$ be a congruence on the semigroup $(P, \otimes)$. The congruence $\theta$ is called an order congruence if there is an order $\lesssim$ on the quotient semigroup $(P / \theta, \otimes)$ such that

(1) $(P / \theta, \lesssim, \otimes)$ is a po-semigroup;

(2) the surjective mapping $\theta^{b}: P \rightarrow P / \theta, x \mapsto \theta^{b}(x)$ is a monotone and multiplicative map. 
Other now it is time to extend to residuated semigroups the notion of quotient. First we give the following definition:

Definition 2.2. Let $(P, \leq, \otimes, \backslash, /)$ be a residuated po-semigroup, $\theta$ be a congruence on the semigroup $(P, \otimes)$. The relation $\theta$ is called a regular congruence if there is an order $\lesssim$ and two binary operations $\backslash$ and / on the quotient algebras $P / \theta$ such that

(1) $(P / \theta, \otimes, \lesssim, \backslash, /)$ is a residuated po-semigroup;

(2) the surjective mapping $\theta^{b}: P \rightarrow P / \theta, x \mapsto \theta^{b}(x)$ is monotone, multiplicative and preserves residuals.

\subsection{Rees congruences on residuated semigroups}

Definition 2.3 ([27]). A non empty subset $I$ of a po-semigroup $\mathbf{P}=(P, \leq, \otimes)$ is called an ideal of $\mathbf{P}$ if (i) $P \otimes I \subseteq I$, (ii) $I \otimes P \subseteq I$. If moreover it satisfies the condition (iii) $x \leq y$ and $y \in I$ imply $x \in I$, then it is called an order ideal of $\mathbf{P}$.

Let $I$ be an ideal of semigroup $(\mathbf{P}, \otimes)$. The Rees congruence $\theta_{I}$ induced by $I$ is equal to $\Delta_{P} \cup(I \times I)$, where $\Delta_{P}=\{(x, x): x \in I\}$. It is equivalently defined by $\theta_{I}=\Delta_{P \backslash I} \cup(I \times I)$. For simplicity, we denote $P / \theta_{I}$ by $P / I$. The factor semigroup $(P / I, \otimes)$ is called Rees factor semigroup. It is clear that $P / I=\{I\} \bigcup\{\{x\}, x \in P \backslash I\}$.

Proposition 2.4 ([27]). Let I be a ideal of po-semigroup P. Then I is a congruence class of an order congruence on $P$ if and only if $I$ is convex, i.e., if $x, y \in I$, then for any $c$ such that $x \leq c \leq y$, it is the case that $c \in I$.

We extend this result to residuated semigroups by introducing the notion of residuated ideal. 
Definition 2.5. A non empty subset of a residuated semigroup $P$ is called a residuated ideal if it satisfies the following axioms (written in deduction form):

(1) $\frac{x, x \backslash y \in I}{y \in I}$ and $\frac{x, y / x \in I}{y \in I}[$ left and right modus Ponens];

(2) $\frac{x \in I, y \in P}{y \backslash x, x / y \in I}[I$ is residually closed $]$.

Theorem 2.6. Let $I$ be a residuated ideal of a residuated semigroup $P$. Then $I$ is a congruence class of a regular congruence on $P$ if and only if $I$ convex.

Proof. Let $I$ be a congruence class for a regular congruence $\theta$. From the proof for 2.4 in [27], we get that $I$ is convex. For the converse, let $\theta_{I}$ be the Rees congruence induced by $I$. As clearly shown in [27], the order $\preccurlyeq$ on the factor algebra $\left(P / \theta_{I}, \otimes\right)$, defined by

$$
\left.\theta_{I}^{b}(x) \preccurlyeq \theta_{I}^{b}(y) \text { iff }(x \leq y) \text { or }\left(x \leq b, b^{\prime} \leq y\right) \text { for some } b, b^{\prime \prime} \in I\right) \text {, }
$$

gives the triple $\left(P / \theta_{I}, \preccurlyeq, \otimes\right)$ a structure of a po-semigroup and the canonical map $\theta_{I}^{b}: P \rightarrow P / \theta_{I}$ is a monotone multiplicative map. It remains to define the residuals on $\left(P / \theta_{I}, \preccurlyeq, \otimes\right)$. For the purpose, we first show that if $x \theta_{I} x^{\prime}$ and $a \theta_{I} a^{\prime}$, then $(a \backslash x) \theta_{I}\left(a \backslash x^{\prime}\right)$ and $(x / a) \theta_{I}\left(x^{\prime} / a^{\prime}\right)$.

Now let's start with $x \theta_{I} x^{\prime}$ and $a \theta_{I} a^{\prime}$. Then $x, x^{\prime} \in I$ or $x=x^{\prime}$; and $a, a^{\prime} \in I$ or $a=a^{\prime}$. We consider four cases.

(i) $x, x^{\prime} \in I$ and $a, a^{\prime} \in I$. From condition (2) of 2.5, $a \backslash x, a \backslash x$ are elements of $I$. The right residual is treated in the same way.

(ii) $x, x^{\prime} \in I$ and $a=a^{\prime}$. Again from the same condition, $a \backslash x, a \backslash x$ are elements of $I$. The right residual is treated in the same way. 
(iii) $x=x^{\prime}$ and $a, a^{\prime} \in I$. In this case, we show that $x / a$ is in $I$ iff $x / a^{\prime}$ is also in $I$ and conversely. Now assume that $x / a \in I$; since $a$ is in $I$, applying Left Modus Ponens, i.e., condition (1) of 2.5, we get that $x$ is in $I$ and so $x / a^{\prime}$ is in $I$. Similarly, if $x / a \in I$, then $x / a^{\prime} \in I$.

(iv) $x=x^{\prime}$ and $a=a^{\prime}$. It is clear that $x / a=x^{\prime} / a^{\prime}$ and $a \backslash x=a \backslash x^{\prime}$.

Now that we have shown that the congruence $\theta_{I}$ is compatible with residuals, one can take as the definition of quotient of residuals

$$
\theta^{b}(y) \backslash \theta^{b}(x):=\theta^{b}(y \backslash x) \text { and } \theta^{b}(x) / \theta^{b}(y):=\theta^{b}(x / y),
$$

and then get the residuated semigroup $(P / \theta, \preccurlyeq, \otimes,(-) \backslash(-),(-) /(-))$ equipped with the projection $\theta^{b}: P \rightarrow P / \theta$, which is monotone, multiplicative and preserves residuals.

\subsection{Characterisation of regular congruences on residuated semigroups}

It turns out that the notion of order congruence (Definition 2.1) can be expressed using a special property of $\theta$.

Proposition 2.7 ([23]). A binary relation on a po-semigroup is an order congruence if and only if for any non negative integer $n$ and for any sequence $a_{1}, a_{2}, \cdots, a_{2 n}$ in $P$,

$$
a_{1} \theta a_{2} \leq a_{3} \theta a_{4} \leq \cdots a_{2 n-1} \theta a_{2 n} \leq a_{1} \Rightarrow \theta^{b}\left(a_{1}\right)=\theta^{b}\left(a_{2}\right)=\cdots=\theta^{b}\left(a_{2 n}\right) .
$$

This proposition extends in extension to regular congruence as defined in Definition 2.2. One can observe that

Proposition 2.8. A regular congruence of a residuated semigroup $\boldsymbol{P}$ is exactly a congruence $\theta$ on the undelying po-semigroup that respects the taking of residuals, i.e., for all $x, y$ and $a$ in Px$\theta y$ implies $(x / a) \theta(y / a)$ and $(x / a) \theta(y / a)$.

We get the following characterization: 
Proposition 2.9. A binary relation on a residuated semigroup is a regular congruence if and only if for any non negative integer $n$ and for any sequence $a_{1}, a_{2}, \cdots, a_{2 n}$ in $P$,

$$
a_{1} \theta a_{2} \leq a_{3} \theta a_{4} \leq \cdots a_{2 n-1} \theta a_{2 n} \leq a_{1} \Rightarrow \theta^{b}\left(a_{1}\right)=\theta^{b}\left(a_{2}\right)=\cdots=\theta^{b}\left(a_{2 n}\right) .
$$

Proof. Assuming $\theta$ to be a regular congruence is just to say that we get the ordered algebra $(P, F, \leq)$ in the sense of [5] where $F=\{\otimes, a \backslash-,-/ a, a \in P\}$. Then we can apply the Proposition 2.1 of [5].

Let $\theta$ be a binary relation on a poset $(P, \leq)$. A $\theta$-chain from an element $x \in P$ to an element $y \in P$ is a finite sequence $x_{1}, x_{1}^{\prime}, x_{2}, x_{2}^{\prime}$, $\ldots, x_{n}, x_{n}^{\prime}$ with

$$
x \leq x_{1} \theta x_{1}^{\prime} \leq x_{2} \theta x_{2}^{\prime} \leq \ldots \leq x_{n} \theta x_{n}^{\prime} \leq y .
$$

We also need to refine the notion of congruence.

Theorem 2.10. Let $\theta$ be a regular congruence on a residuated semigroup $P$.

(i) The relation $\preccurlyeq_{\theta}$ on $P$ as follows

$$
x \preccurlyeq_{\theta} y \text { iff there exists } \theta \text {-chain from } x \text { to } y
$$

is a compatible preorder, i.e., it a reflexive and transive relation compatible with the order.

(ii) The relation $v(\theta)=\preccurlyeq_{\theta} \cap \preccurlyeq_{\theta}^{0}$ is a regular congruence on $P$. It is regular congruence generated by $\theta$.

Proof. That the relation $\preccurlyeq$ is a preorder is proved exactly as in [23] (see proof of Theorem 5). It remains to check the compatibility with taking the residuals. If $x \leq x_{1} \theta x_{1}^{\prime} \leq x_{2} \theta x_{2}^{\prime} \leq \ldots \leq x_{n} \theta x_{n}^{\prime} \leq y$ is a $\theta$-chain from $x$ to $y$, then the sequence $(a \otimes x) \leq\left(a \otimes x_{1}\right) \theta\left(a \otimes x_{1}^{\prime}\right) \leq\left(a \otimes x_{2}\right)$ $\theta\left(a \otimes x_{2}^{\prime}\right) \leq \cdots \leq\left(a \otimes x_{n}\right) \theta\left(a \otimes x_{n}^{\prime}\right) \leq(a \otimes y)$ is a $\theta$-chain from $a \otimes x$ to 
$a \otimes y$ and the sequence $(x \otimes a) \leq\left(x_{1} \otimes a\right) \theta\left(x_{1}^{\prime} \otimes a\right) \leq\left(x_{2} \otimes a\right) \theta\left(x_{2}^{\prime} \otimes a\right)$ $\leq \cdots \leq \theta\left(x_{n}^{\prime} \otimes a\right) \leq(y \otimes a)$ is a $\theta$-chain from $x \otimes a$ to $y \otimes a$; this comes from the fact that $\leq$ and $\theta$ are compatible with the multiplication. Similarly, one gets a $\theta$-chain from $a \backslash x$ to $a \backslash y$ and a $\theta$-chain from $x / a$ to $y / a$ by dividing on the left or on the right by $a$; this follows from the fact that $\theta$ is compatible with the taking of the residuals and that the residuals $a \backslash-$ and $-/ a$ are monotone maps.

The rest of the proof is a direct rewriting of proof of Theorem 5 of [23] but now using Proposition 2.9.

The last proposition can be used to characterise the regular congruence on a residuated semigroup generated by a Rees congruence induced by a residuated lattice.

Theorem 2.11 (The regular congruence generated by a residuated ideal).

Let $I$ be residuated ideal of $P$. The regular congruence $R_{I}$ generated by $I$ is described as follow $x R_{I} y$ iff $x=y$ or there exist $b, b^{\prime}, c, c^{\prime} \in I$ such that $x \leq b, b^{\prime} \leq y$ and $y \leq c, c^{\prime} \leq x$. The order on $P / v\left(\theta_{I}\right)$ is given by

$$
\theta_{I}^{b}(x) \preccurlyeq \theta_{I}^{b}(x) \Leftrightarrow(x \leq y) \text { or }\left(x \leq a, a^{\prime} \leq y \text {, for some } a, a^{\prime} \in I\right) \text {. }
$$

Proof. If we follow the proof of Theorem 6 of [23], it is enough to check that the binary relation $v\left(\theta_{I}\right)$ is compatible with taking the residuals, which is actually true since the maps $a \backslash-$ and $-/ a$ are increasing maps.

\section{Acknowledgement}

The authors would like to thank an anonymous referee for the valuable suggestions and corrections. 


\section{References}

[1] S. L. Bloom, Varieties of ordered algebras, J. Compt. System Sci. 13(2) (1976), 200-212.

DOI: https://doi.org/10.1016/S0022-0000(76)80030-X

[2] T. S. Blyth and M. F. Janowitz, Residuation Theory, Pergamon Press, 1972.

[3] F. Borceux, Handbook of Categorical Algebra, Vol. 2, Encyclopedia of Mathematics and its Applications, 51, Cambridge University Press, Cambridge, 1994.

[4] R. L. Cignoli, I. M. d'Ottaviano and D. Mundici, Algebraic Foundations of ManyValued Reasoning, Kluwer Dordrecht, 2000.

[5] G Czédli and A. Lenkehegyi, On congruence $n$-distributivity of ordered algebras, Acta Math. Hungar. 41(1-2) (1983), 17-26.

DOI: https://doi.org/10.1007/BF01994057

[6] B. A. Davey and H. A. Priestley, Introduction to Lattices and Order, Cambridge University Press, 1980.

[7] M. Erné, Adjunctions and Galois connections: Origin, History and Development, in K. Denecke, M. Erné and S. L. Wismath (Eds.), Galois Connections and Applications, Kluwer Academic Publishers, Dordrecht-Boston, 2004, pp. 1-138.

[8] M. Erné, J. Koslowski, A. Melton and G. E. Strecker, A primer on Galois connections, Annals of the New York Academy of Sciences 704(1) (1999), 103-125

DOI: https://doi.org/10.1111/j.1749-6632.1993.tb52513.x

[9] N. Galatos, P. Jipsen, T. Kowalski and H. Ono, Residuated Lattices: An Algebraic Glimpse at Sub-Structural Logics, Elsevier, 2007, 532 pp.

[10] R. Goldblatt, A Kripke-Joyal Semantics for noncommutative logic in quantales, pp 209-225 in Governatori, Hodkinson and Venema (Eds.), Advances in Modal Logic, Volume 6, College Publications, London, 2006.

[11] P. Hajek, Metamathematics of Fuzzy Logic, Kluwer Academic Publishers, Dordrecht, 1998.

DOI: https://doi.org/10.1007/978-94-011-5300-3

[12] P. T. Johnstone, Stone Spaces, Cambridge University Press, Cambridge, 1982.

[13] Kimmo I. Rosenthal, Quantales and their Applications, Pitman Res. Notes in Math. Series 234, Longman, 1990.

[14] C. J. Mulvey, “ $6 \zeta$ ” Rendiconti Circ. Mat. Palermo 12 (1986), 99-104.

[15] Susan B. Niefield and Kimmo I. Rosenthal, Constructing locales from quantales, Mathematical Proceedings of the Cambridge Philosophical Society 104(2) (1988), 215-234.

DOI: https://doi.org/10.1017/S0305004100065403 
[16] Niovi Kehayopulu and Michael Tsingelis, On free ordered semigroups, Scientiae Mathematicae Japonicae, Online (e-2010), 97-103.

[17] H. Ono, Semantics for substructural logics, In: Substructural Logics, eds. by P. Schroeder-Heister and K. Dosen, Oxford University Press (1993), 259-291.

[18] H. Ono, Structural rules and a logical hierarchy, in: Mathematical Logic, Proceedings of the Summer School and Conference on Mathematical Logic, Heyting 88, P. P. Petrov (Editor), Plenum Press (1990), 95-104.

[19] H. Ono and Y. Komori, Logics without the contraction rule, Journal of Symbolic Logic 50(1) (1985), 169-201.

DOI: https://doi.org/10.2307/2273798

[20] A Ogus, Lectures on Logarithmic Algebraic Geometry, University of California, Berkeley, 2006.

[21] Sergey A. Solovyov, A note on nuclei of quantale algebras, Bulletin of the Section of Logic 40(1-2) (2011), 91-112.

[22] H. Simmons, An Algebraic Version of Cantor Bendixson Analysis, In Categorical Aspects of Topology and Analysis (Ottawa, Ont., 1980), pages 310-323, Springer, Berlin, 1982.

[23] Shi Xian Ping and Xie Xiang Yun, A note on order congruences on ordered semigroups, Journal of Mathematical Research \& Exposition 28(4) (2008), 898-904.

[24] H. Simmons, Near-discreteness of modules and spaces as measured by Gabriel and Cantor, J. Pure Appl. Algebra 56(2) (1989), 119-162.

DOI: https://doi.org/10.1016/0022-4049(89)90141-2

[25] Valdis Laan and Sohhail Nasir, On monomorphisms and epimorphisms in varieties of ordered algebras, Communication in Algebra 43(7) (2015), 2802-2819.

DOI: https://doi.org/10.1080/00927872.2014.904328

[26] M. Ward and R. P. Dilworth, Residuated lattices, Trans. Amer. Math. Soc. 45(3) (1939), 335-354.

DOI: https://doi.org/10.1090/S0002-9947-1939-1501995-3

[27] Xie Xiang-Yun, On regular, strongly regular congruences on ordered semigroups, Semigroup Forum 61(2) (2000), 159-178.

DOI: https://doi.org/10.1007/PL00006018 